\title{
The Effects and the Mechanisms of Sodium Glucose Cotransporter-2 Inhibition in Heart Failure
}

ISSN: 2578-0263

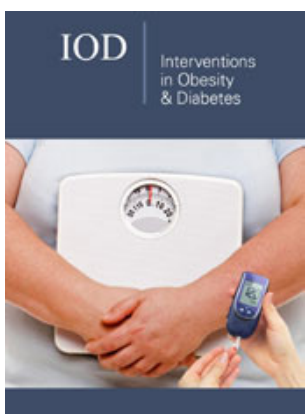

*Corresponding author: Hui Gong, Department of Cardiology, Jinshan Hospital of Fudan University, 1508 Longhang Road, Jinshan District, Shanghai, 201508, China, E-mail address: 13918566212@163.com (H Gong)

Submission: 佂 February 13, 2020

Published: 此 February 26, 2020

Volume 3 - Issue 5

How to cite this article: Lei zhou, Hui Gong. The Effects and the Mechanisms of Sodium Glucose Cotransporter-2 Inhibition in Heart Failure. Interventions Obes Diabetes 3(5). IOD.000571.2020. DOI: $10.31031 /$ IOD.2020.03.000571

Copyright@ Hui Gong. This article is distributed under the terms of the Creative Commons Attribution 4.0 International License, which permits unrestricted use and redistribution provided that the original author and source are credited.

\author{
Lei zhou and Hui Gong* \\ Department of Cardiology, Jinshan Hospital of Fudan University, Shanghai, 201508, China
}

\begin{abstract}
Although treatments are currently established, heart failure (HF) remains the leading cause of hospitalization and mortality worldwide. Therefore, new therapeutic targets are needed to improve the prognosis of patients with heart failure, especially those with preserved ejection fraction. In recent years, many clinical studies have shown that sodium glucose cotransporter-2inhibitors can significantly reduce the hospitalization rate and mortality of heart failure. It is expected to become a new method for the treatment of heart failure, and this effect also exists in non-diabetic heart failure patients. But its potential mechanisms of action are not fully understood yet and needs further exploration. This article reviews the effects and the potential mechanisms of SGLT2 inhibitors in heart failure,
\end{abstract}

Keywords: SGLT2 inhibitors; Heart failure

\section{Introduction}

Individuals with diabetes are not only with high risk of developing heart failure but also with increased risk of death [1]. Different types of hypoglycemic drugs have different cardiovascular safety, so it is necessary to evaluate the cardiovascular safety of new hypoglycemic drugs. In recent years, many large-scale randomized controlled trials on SGLT2 inhibitors for oral hypoglycemic agents such as (EMPA-REG OUTCOME (The Empagliflozin, Cardiovascular Outcomes, and Mortality in Type 2 Diabetes Trial); CANVAS Program (The Canagliflozin Cardiovascular Assessment Study Program); DEGLARE-TIMI 58 (The Dapagliflozin Effect on Cardiovascular Events -TIMI 58 Trial); DEFINE-HF Trial (The Dapagliflozin and Prevention of Adverse Outcomes in Heart Failure Trial) have shown that SGLT2 inhibitors could significantly reduce the hospitalization rate and the composite endpoint of cardiovascular mortality in patients with heart failure compared to the placebo group [2-5]. This effect persisted even in non-diabetic patients. The mechanisms responsible for the cardioprotective effects of SGLT2 inhibitors remain uncertain. This article summarizes the possible mechanisms of SGLT2 inhibitors in heart failure as follows.

\section{The Biological Role of SGLT2}

Sodium-glucose co-transporters (SGLTs) belong to the SLC5 family of active glucose transporters with 12 members. Within the family, SGLT1 and SGLT2 have received the most attention in medicine. SGLT-1 is mainly expressed on the brush-border membrane of the small intestine and the straight (S3 segment) renal proximal tubule, whereas SGLT-2 is mainly expressed on the apical membrane of renal proximal convoluted tubules (S1 and S2 segments) [6]. SGLT2 is a low-affinity, high-capacity glucose transporter that accounts for about $90 \%$ of the reabsorption of filtered glucose by the kidney, and the remaining $10 \%$ is completed by SGLT-1 $[7,8]$. In contrast to traditional hypoglycemic drugs, SGLT2 inhibitors lower plasma glucose levels through competitively binding to sodium glucose cotransporter- 2 in the kidney, thereby reducing the reabsorption of glucose and sodium by the kidney. As a result, it can produce a range of beneficial systemic effects, including reduced blood glucose levels, lower blood pressure, sodium diuresis, osmotic diuresis, renal and cardiac protection [9].

\section{The Mechanism of SGLT2 Inhibitor in Heart Failure}

\section{SGLT2 inhibitor improves myocardial metabolic remodeling}

Substrates for normal cardiac energy metabolism include fatty acids and sugars, lactic acid, branched chain amino acids, and ketone bodies, with fatty acid oxidation as the main substrate. During heart failure, due to myocardial hypoxia, the heart undergoes metabolic 
remodeling, and myocardial fatty acid oxidation is significantly reduced. Myocardial substrate oxidation switches from fat to carbohydrate oxidation (fetal-like metabolism) [10]. Accumulating reports have demonstrated that SGLT-2 inhibitors could promote the degradation of fatty acids, glucose, branched-chain amino acids, and ketone bodies during heart failure, resulting in improved myocardial energetics and efficiency. Santos-Gallego et al. found that the SGLT2 inhibitors empagliflozin could increase the oxidation of ketone bodies, branched chain amino acids, fatty acids and related metabolic enzymes expression in the myocardium in a heart failure pig model, thereby improving myocardial energy metabolism and enhancing ventricular systolic function and ameliorating adverse left ventricular remodeling [11]. In the mouse model of diabetes, the SGLT2 inhibitor empagliflozin has been shown to increase the total cardiac ATP production by $30 \%$ through increasing the oxidation rate of glucose and fatty acids [12]. Yurista et al. [13] reported that SGLT-2 inhibitor empagliflozin improved cardiac function in nondiabetic rats with left ventricular dysfunction after myocardial infarction through increasing the oxidation of ketone bodies [13]. It has been shown that a single dose of empagliflozin enhanced cardiac energy status by increasing plasma ketone levels [14].

\section{SGLT2 inhibitor improves myocardial remodeling}

Ventricular remodeling is the basic pathophysiological process of the occurrence and development of heart failure. Several studies have demonstrated that SGLT2 inhibitors could alleviate ventricular remodeling. Zhang et al. reported that SGLT2 inhibitors dapagliflozin could reverse the left ventricle's concentric remodeling by reducing aortic sympathetic tone activity and aortic inflammation in a pig model of heart failure with preserved ejection fraction [15]. Myocardial fibrosis is also an important pathological feature of myocardial remodeling. It has been shown that the SGLT2 inhibitors empagliflozin could attenuate myocardial fibrosis and ventricular remodeling by inhibiting transforming growth factor $\beta /$ Smad signaling pathway in a mouse model of diabetes [16], Lee et al. [17] showed that in infarcted rat hearts, the SGLT2 inhibitors dapagliflozin promoted the differentiation of macrophages into M2 anti-inflammatory types by inhibiting the STAT3 signaling pathway, while M2 macrophages inhibit fibroblasts toward myofibroblast cell differentiation to reduce myocardial fibrosis [17]. Myocardial fibrosis that was observed in obese and type 2 diabetic mice was alleviated after empagliflozin treatment through reducing cardiac macrophage infiltration [18]. Moreover, empagliflozin has been shown to exhibit improved atrial and ventricular remodeling in hypertensive heart failure rats [19].

\section{SGLT2 inhibitor improves sympathetic nerve activity}

Excessive activation of the sympathetic nervous system (SNS) plays an important role in the occurrence and development of heart failure, leading to an increase in cardiac load and atrial/ventricular remodeling. Studies have shown that the use of SGLT2 inhibitors could reduce aortic blood pressure without causing compensatory changes in heart rate. Therefore, it was speculated that SGlT2 inhibitors might improve heart failure by generating sympathetic inhibitory effects [20], Kiuchi et al. [21] reported that long-term use of the SGLT2 inhibitor empagliflozin ameliorated cardiac sympathetic nerve activity in a patient with heart failure [21]. In a pig model of heart failure with preserved ejection fraction, the aortic sympathetic nerve activity was attenuated after dapagliflozin treatment through downregulating the expression of tyrosine hydroxylase in the aorta [15].

\section{SGLT2 inhibitors regulate calcium homeostasis in cardiomyocytes}

SERCA2a is a calcium ion ATPase on the sarcoplasmic network of cardiomyocytes. Its activity is regulated by phospholamban. During the diastole of cardiomyocytes, it can excrete calcium ions from the cytoplasm into the sarcoplasmic network to promote myocardial relaxation. In cardiomyocytes of heart failure, SERCA2a activity is significantly reduced, accompanied by $\mathrm{Ca}^{2+}$ transport disorder, which is manifested by diastolic sarcoplasmic reticulum intake of $\mathrm{Ca}^{2+}$ and accumulation of intracellular $\mathrm{Ca}^{2+}$, which affects the diastolic function of the heart; while systolic calcium release decreases, $\mathrm{Ca}^{2+}$ decreases in cytoplasm, and systolic function decreases accordingly $[22,23]$. It has been shown that empagliflozin improved LV diastolic function by increasing sarcoplasmic endoplasmic reticulum $\mathrm{Ca}^{2+}$-ATPase (SERCA2a) activity in genetic diabetic mice [24]. Joubert et al. [25] also found that dapagliflozin enhanced SERCA2a activity leading to improved cardiac contractile dysfunction in a diabetic lipodystrophic mouse model [25].

\section{SGLT2 inhibitor improves tissue cell hypoxia}

Tissue cells are often hypoxic during heart failure. Studies have found that SGLT2 inhibitor empagliflozin could increase the concentration of erythropoietin and hematocrit in plasma, promote the production of red blood cells and hemoglobin, improve tissue hypoxia, and ameliorate heart failure [26]. A COX regression analysis of a large randomized controlled clinical trial of englitazone also showed that hematocrit and increased hemoglobin may be the main reasons for mediating the cardiovascular effects of empagliflozin [27]. Therefore, SGLT2 inhibitors may improve heart failure by increasing oxygen supply to tissue cells throughout the body.

\section{SGLT2 inhibitors inhibit the activity of the sodium- hydrogen exchanger NHE}

Sodium hydrogen exchange protein (NHE) is responsible for the exchange of sodium ions and hydrogen ions on the cell membrane to maintain the electrolyte distribution. NHE is involved in the occurrence and development of heart failure and myocardial hypertrophy, and inhibition of NHE activity can prevent and ameliorate heart failure [28,29]. Studies have observed that the SGLT2 inhibitors empagliflozin, canagliflozin, and dapagliflozin could inhibit the activity of NHE, reduce the sodium and calcium concentrations in the cytoplasm of myocardial cells, and increase 
the calcium ion concentration in mitochondria [30,31]. Therefore, some scholars speculated that SGLT2 inhibitors might ameliorate heart failure by inhibiting the activity of NHE to regulate the ion imbalance of myocardial cells during heart failure.

\section{SGLT2 inhibitors reduce cardiac preload and afterload}

Myocardial cells are often hypoxic during heart failure. The oxygen consumption of cardiomyocytes is related to cardiac workload and heart rate. The SGLT2 inhibitors reduce preload through sodium diuresis and osmotic diuresis [32]. SGLT2 inhibitors reduce blood pressure and arterial stiffness without increasing heart rate, thereby decreasing cardiac afterload [33]. Several studies have revealed that SGLT-2 inhibitors (dapagliflozin; canagliflozin empagliflozin) could lead to a considerate reduction in arterial stiffness in T2DM patients [34-36]. This effect has been attributed to improved endothelial dysfunction $[15,36]$. It was hypothesized that the sustained reduction in intravascular volume and blood pressure may lead to a reduction in cardiac preload and afterload, respectively, thereby alleviating cardiac workload and improving cardiac function [32].

\section{SGLT2 inhibitor improves arterial stiffness and endothelial cell function}

Several researches have reported significant reduction in arterial stiffness in patients with T2DM treated with empagliflozin, canagliflozin, and dapagliflozin. This effect was related to the improvement of endothelial dysfunction by SGLT2 inhibitors. Reduced arterial stiffness and improved endothelial function could lead to decreased blood pressure, reduce cardiac load, improve myocardial cell hypoxia, and ameliorate heart failure $[15,34$ 36]. In addition, Zhang et al. [15] found that the SGLT2 inhibitor dapagliflozin improved endothelial cell function by upregulating the NO-cGMP-PKG signaling pathway and inhibiting the inflammatory response of endothelial cells.

\section{Conclusion}

Current clinical trial studies of SGLT-2 inhibitors demonstrate beneficial effects on heart failure in non-diabetic patients. A clinical trial of the SGLT2 inhibitor dapagliflozin included 4,744 patients with chronic heart failure and reduced ejection fraction $(\mathrm{EF}<45 \%)$. Diabetes was neither an inclusion nor exclusion criterion in this study. After 1.5 years of treatment and follow-up, the study indicated that the SGLT2 inhibitor dagliflozin reduced the risk of exacerbating heart failure by $30 \%$ and the risk of cardiovascular death by $18 \%$ compared to the placebo group [5]. In summary, SGLT2 inhibitors are expected to become a new class of drugs for treating heart failure. The beneficial effects of SGLT2 inhibitors on heart failure maybe by improving ventricular remodeling, improving myocardial metabolic remodeling, reducing autonomic nerve activity, improving myocardial cell calcium homeostasis, inhibiting NHE activity and so on. But a large number of basic and clinical trials are still needed to prove the feasibility of SGLT2 inhibitors in the treatment of heart failure. In the future, SGLT-2 inhibitors may be used to treat heart failure in patients with nontype 2 diabetes.

\section{References}

1. Gilbert RE, Krum H (2015) Heart failure in diabetes: Effects of antihyperglycaemic drug therapy. Lancet 385(9982): 2107-2117.

2. Zinman B, Wanner C, Lachin JM, Fitchett D, Bluhmki E, et al. (2015) Empagliflozin, cardiovascular outcomes, and mortality in type 2 diabetes. N Engl J Med 373(22): 2117-2128.

3. Wiviott SD, Raz I, Bonaca MP, Mosenzon O, Kato ET, et al. (2019) Dapagliflozin and cardiovascular outcomes in type 2 diabetes. N Engl J Med 380(4): 347-357.

4. Radholm K, Figtree G, Perkovic V, Solomon SD, Mahaffey KW, et al. (2018) Canagliflozin and heart failure in type 2 diabetes mellitus. Circulation 138(5): 458-468.

5. Mcmurray JJV, Solomon SD, Inzucchi SE, Køber L, Kosiborod MN, et al. (2019) Dapagliflozin in patients with heart failure and reduced ejection fraction. N Engl J Med 381(21): 1995-2008.

6. Ferrannini E (2017) Sodium-glucose co-transporters and their inhibition: Clinical physiology. Cell metabolism 26(1): 27-38.

7. Diamant M, Morsink LM (2013) SGLT2 inhibitors for diabetes: Turning symptoms into therapy. Lancet 382(9896): 917-918.

8. Hummel CS, Lu C, Loo DD, Hirayama BA, Voss AA, et al. (2011) Glucose transport by human renal Na+/D-glucose cotransporters SGLT1 and SGLT2. American journal of physiology Cell physiology 300(1): C14-C21.

9. Scheen A J (2015) Pharmacodynamics, efficacy and safety of sodiumglucose co-transporter type 2 (SGLT2) inhibitors for the treatment of type 2 diabetes mellitus. Drugs 75(1): 33-59.

10. Van Bilsen M, Smeets PJ, Gilde AJ, van der Vusse GJ (2004) Metabolic remodeling of the failing heart: The cardiac burn-out syndrome? Cardiovasc Res 61(2): 218-226.

11. Santos Gallego CG, Requena Ibanez JA, San Antonio R, Ishikawa K, Watanabe S, et al. (2019) Empagliflozin ameliorates adverse left ventricular remodeling in nondiabetic heart failure by enhancing myocardial energetics. J Am Coll Cardiol 73(15): 1931-1944.

12. Verma S, Rawat S, Ho KL, Wagg CS, Zhang L, et al. (2018) Empagliflozin increases cardiac energy production in diabetes: Novel translational insights into the heart failure benefits of SGLT2 inhibitors. JACC Basic to translational science 3(5): 575-587.

13. Yurista SR, Sillje HHW, Oberdorf Maass SU, Schouten EM, Pavez Giani MG, et al. (2019) Sodium-glucose co-transporter 2 inhibition with empagliflozin improves cardiac function in non-diabetic rats with left ventricular dysfunction after myocardial infarction. Eur J Heart Fail 21(7): 862-873.

14. Abdurrachim D, Manders E, Nicolay K, Mayoux E, Prompers JJ (2018) Single dose of empagliflozin increases in vivo cardiac energy status in diabetic db/db mice. Cardiovasc Res 114(14): 1843-1844.

15. Zhang N, Feng B, Ma X, Sun K, Xu G, et al. (2019) Dapagliflozin improves left ventricular remodeling and aorta sympathetic tone in a pig model of heart failure with preserved ejection fraction. Cardiovascular diabetology 18(1): 107.

16. Li C, Zhang J, Xue M, Li X, Han F, et al. (2019) SGLT2 inhibition with empagliflozin attenuates myocardial oxidative stress and fibrosis in diabetic mice heart. Cardiovascular diabetology 18(1): 15.

17. Lee TM, Chang NC, Lin SZ (2017) Dapagliflozin, a selective SGLT2 Inhibitor, attenuated cardiac fibrosis by regulating the macrophage polarization via STAT3 signaling in infarcted rat hearts. Free Radical Biology and Medicine 104: 298-310.

18. Lin B, Koibuchi N, Hasegawa Y, Sueta D, Toyama K, et al. (2014) Glycemic control with empagliflozin, a novel selective SGLT2 inhibitor, ameliorates cardiovascular injury and cognitive dysfunction in obese and type 2 diabetic mice. Cardiovascular diabetology 13: 148. 
19. Lee HC, Shiou YL, Jhuo SJ, Chang CY, Liu PL, et al. (2019) The sodiumglucose co-transporter 2 inhibitor empagliflozin attenuates cardiac fibrosis and improves ventricular hemodynamics in hypertensive heart failure rats. Cardiovascular diabetology 18(1): 45.

20. Wan NN, Rahman A, Hitomi H, Nishiyama A (2018) The effects of sodium-glucose cotransporter 2 inhibitors on sympathetic nervous activity. Frontiers in endocrinology 9: 421.

21. Kiuchi S, Hisatake S, Kabuki T, Fujii T, Oka T, et al. (2018) Long-term use of ipragliflozin improved cardiac sympathetic nerve activity in a patient with heart failure: A case report . Drug discoveries \& therapeutics 12(1): 51-54.

22. Kawase Y, Hajjar RJ (2008) The cardiac sarcoplasmic/endoplasmic reticulum calcium ATPase: A potent target for cardiovascular diseases . Nat Clin Pract Cardiovasc Med 5(9): 554-565.

23. Wei J, Liu HC, Lee FY, Lee Ms, Huang CY, et al. (2003) Role of the sarcoplasmic reticulum in altered action potential and contraction of myopathic human and hamster ventricle. Clinical and experimental pharmacology \& physiology 30(4): 232-241.

24. Hammoudi N, Jeong D, Singh R, Farhat A, Komajda M, et al. (2017) Empagliflozin improves left ventricular diastolic dysfunction in a genetic model of type 2 diabetes. Cardiovascular drugs and therapy 31(3): 233246.

25. Joubert M, Jagu B, Montaigne D, Marechal X, Tesse A, et al. (2017) The sodium-glucose cotransporter 2 inhibitor dapagliflozin prevents cardiomyopathy in a diabetic lipodystrophic mouse model . Diabetes 66(4): 1030-1040.

26. Mazer CD, Hare GMT, Connelly PW, Gilbert RE, Shehata N, et al. (2019) Effect of empagliflozin on erythropoietin levels, iron stores and red blood cell morphology in patients with type 2 diabetes and coronary artery disease. Circulation 141(8): 704-707.

27. Inzucchi SE, Zinman B, Fitchett D, Wanner C, Ferrannini E, et al. (2018) How does empagliflozin reduce cardiovascular mortality? insights from a mediation analysis of the EMPA-REG OUTCOME trial. Diabetes care 41(2): 356-363.
28. Nakamura TY, Iwata Y, Arai Y, Komamura K, Wakabayashi S (2008) Activation of $\mathrm{Na}+\mathrm{H}+$ exchanger 1 is sufficient to generate $\mathrm{Ca} 2+$ signals that induce cardiac hypertrophy and heart failure. Circulation Research 103(8): 891-893.

29. Engelhardt S, Hein L, Keller U, Klämbt K, Lohse MJ (2002) Inhibition of $\mathrm{Na}(+)-\mathrm{H}(+)$ exchange prevents hypertrophy, fibrosis, and heart failure in beta(1)-adrenergic receptor transgenic mice. Circulation Research 90(7): 814-819.

30. Uthman L, Baartscheer A, Bleijlevens B, Schumacher CA, Fiolet JWT, et al. (2018) Class effects of SGLT2 inhibitors in mouse cardiomyocytes and hearts: Inhibition of $\mathrm{Na}^{+} / \mathrm{H}^{+}$exchanger, lowering of cytosolic $\mathrm{Na}^{+}$and vasodilation . Diabetologia 61(3): 722-726.

31. Baartscheer A, Schumacher CA, Wust RC, Fiolet JW, Stienen GJ, et al. (2017) Empagliflozin decreases myocardial cytoplasmic $\mathrm{Na}^{+}$through inhibition of the cardiac $\mathrm{Na}^{+} / \mathrm{H}^{+}$exchanger in rats and rabbits . Diabetologia 60(3): 568-573.

32. Sattar N, Mclaren J, Kristensen SL, Preiss D, McMurray JJ (2016) SGLT2 Inhibition and cardiovascular events: why did EMPA-REG Outcomes surprise and what were the likely mechanisms? Diabetologia 59(7): 1333-1339.

33. Scheen AJ (2019) Effect of SGLT2 inhibitors on the sympathetic nervous system and blood pressure. Current Cardiology Reports 21(8): 70.

34. Ramirez AJ, Sanchez MJ, Sanchez RA (2019) Diabetic patients with essential hypertension treated with amlodipine: Blood pressure and arterial stiffness effects of canagliflozin or perindopril. J Hypertens 37(3): 636-642

35. Chilton R, Tikkanen I, Cannon CP, Crowe S, Woerle HJ, et al. (2015) Effects of empagliflozin on blood pressure and markers of arterial stiffness and vascular resistance in patients with type 2 diabetes. Diabetes Obesity \& Metabolism 17(12): 1180-1193.

36. Solini A, Giannini L, Seghieri M, Vitolo E, Taddei S, et al. (2017) Dapagliflozin acutely improves endothelial dysfunction, reduces aortic stiffness and renal resistive index in type 2 diabetic patients: A pilot study. Cardiovascular diabetology 16(1): 138. 